\title{
Simulating heart rate kinetics during incremental and interval training
}

\author{
Maria S. Zakynthinaki ${ }^{1,2}$ \\ 1 Department of Electronics, School of Applied Sciences, University of Applied Sciences, Crete, Greece; ${ }^{2}$ Applied \\ Mathematics and Computers Laboratory, Technical University of Crete, Chania, Greece
}

\section{Summary}

Study aim: To apply a recently proposed dynamical systems model to simulate, for the first time, the heart rate (HR) response to exercise of time-dependent intensity.

Material and methods: The applied model is expressed in the form of two coupled ordinary differential equations, one giving the rate of change of $H R$ and the other providing the time dependency of exercise intensity. According to the model, the $H R$ is assumed to depend on time, velocity, lactate accumulation and the subject's overall cardiovascular condition. For the purposes of the present work, training sessions were simulated, consisting of exponentially and step-wise increasing intensities, as well as interval training.

Results: Training sessions of exponentially and step-wise increasing velocity, as well as an interval training session, have been simulated. Successful simulations of the corresponding $H R$ response were achieved.

Conclusions: The present work successfully demonstrates the model's excellent performance in simulating the HR response to exercises of time-dependent intensity. The applied model has been shown to correctly simulate the heart rate response also during exercises of complicated intensity patterns, such as the interval training session. The study confirms the ability of the implemented mathematical model not only to simulate and predict heart rate kinetics for any given exercise intensities but also to provide important information regarding an individual's cardiovascular condition. This is of vast importance, not only in the area of fitness and sport, where it can serve as a fundamental tool for the design of efficient training sessions, but also in the areas of cardiovascular health, prediction and rehabilitation.

Keywords: Modelling - Simulation - Exercise physiology

\section{Introduction}

Based on ventricle contractions, heart rate is defined as the number of heart beats per minute. The temporal evolution of the heart rate (heart rate kinetics) of a person who starts moving from rest depends on the intensity of the exercise, as well as on a number of other factors, such as temperature, heat, fatigue, age, over-training, nutrition and hydration, altitude, medication, infectious disease or even mental activity $[1,8,24]$.

The analysis of the heart rate response to exercise is a very important tool in the areas of physiology, cardiovascular health, prediction and rehabilitation, as it can detect hidden physiological responses or abnormalities and also provide important information regarding cardiovascular condition (see also [1, 2, 3, 9, 11, 13, 22]). In the area of fitness and sport it can also serve as a fundamental tool for the design of efficient training sessions.
Two important values are associated with heart rate: its maximum value $H R_{\max }$, which is the highest value that can be achieved in an all-out effort to the point of exhaustion, and its resting value $H R_{\text {min }}$, which is measured in the absence of any movement while the subject is relaxed but awake, in a neutrally temperate environment, not having recently exerted themselves or having been subject to stress or surprise $[8,14,17,24]$. Professional athletes have been reported to have a resting heart rate as low as 35 beats/min, while for a normal person of age 10 years or more it is normally 60 beats/min $<H R \min <100$ beats/ $\min [1,9,24,27]$. Men have been shown to have a lower resting heart rate than women, with a reported difference of about 5 beats/min in athletes [24]. Regarding its maximum value $H R_{\max }$, it should be noted that this is a highly reliable value that remains constant for a particular subject [25] and changes only slightly with age (a slight but steady decrease of about one beat/min per year, beginning at 10 to 15 years of age, has been observed). 
The heart rate of an individual increases or decreases between these two extreme values in response to the body's need to maintain an equilibrium between requirement and delivery of oxygen and nutrients. Changes in heart rate can be provoked by physical exercise, fatigue (lactate accumulation in the blood), changes in temperature, sleep, nutrition, hydration, altitude, medication, diseases, anxiety or stress $[1,8,24]$.

When all other parameters that can affect the heart rate during exercise are kept constant, then the heart rate response can be assumed to be a function of exercise intensity and the individual's cardiovascular condition. The "heart rate demand" $D$, which is the value the heart rate is called to meet, depends in general on time and exercise intensity. Always $D \geq H R_{\min }$. For low intensity exercises the demand $D$ is only intensity-dependent and $D<H R_{\max }$. For severe or very high intensity exercise $D \geq H R_{\max }$ and an abrupt rise in heart rate towards $H R_{\max }$ is observed [24]. For such exercise intensities the achievement of a steady state in the heart rate values is delayed and a slow increase in heart rate can be observed, a phenomenon that has been referred to as the slow component [21, 24].

Various models of the cardiovascular response to exercise have been proposed, mainly based on the statistical goodness of fit of the model curves to heart rate data, starting from the mono-exponential model for moderate constant exercise intensity [11] and continuing to the threephase model $[2,3,9,13,18,20,22]$, which gives the heart rate response to constant intensity exercise by means of three exponential functions. Other models using first order lineal approximations have also been proposed (see [15] and the references therein); however, the implementation of these models is limited, as they can only be applied to exercises of constant intensity.

The dynamical systems model presented in [20], which is based on a similar model of oxygen uptake kinetics $[19,21]$, simulates and predicts the heart rate response to exercise via numerical solution of a system of coupled differential equations, one giving the rate of change of heart rate and the other giving the rate of change of exercise velocity. This model can be implemented for exercise of linear or non-linear intensities, of any functional form. Another recently proposed model of heart rate dynamics during cycling [15] is based on the dynamical systems model of [20] and simulates heart rate dynamics across non-linear exercise intensities by introducing a differential equation for the rate of change of heart rate demand. According to this model, the rate of change of $D$ is assumed to depend on the current value of $D$, as well as the power output and cadence, and two more model parameters are introduced.

Based on the assumption that a model should include as few parameters as possible, an improved and advanced model of [20] has also been recently proposed [24]. This model assumes only one parameter, which is related to the subject's overall cardiovascular condition and takes into account the dynamics of the body's physiological responses, i.e. lactate accumulation and the kinetics near the extremes, the maximum and minimum heart rate values.

The aim of the present study is to apply this recently proposed dynamical systems model [24] to simulate, for the first time, the heart rate $(H R)$ response to exercise of time-dependent intensity.

\section{Materials and methods}

\section{Modelling heart rate kinetics as a dynamical system}

This section presents a brief introduction to the dynamical systems model herein implemented for the simulation of heart rate kinetics. For more details on this model please refer to [24].

The model is expressed in the following form of a system of coupled ordinary differential equations [24], one regarding the rate of change of heart rate $H R$ and the other regarding the rate of change of exercise intensity (velocity $v)$ :

$$
\begin{aligned}
\dot{H} R & =-f_{\min } f_{\max } f_{D} \\
\dot{v} & =I(t)
\end{aligned}
$$

where:

- $t$ is the time elapsed from the beginning of the data recording session,

- $\dot{H} R=\frac{\mathrm{d} H R}{\mathrm{~d} t}$,

- $\quad v$ is the exercise velocity, which is in general time-dependent,

- $\lambda \in[0,1]$ is a global parameter of the model that defines fitness level, such that $\lambda \approx 1$ refers to an excellent cardiovascular condition,

- $H R \equiv H R(H R(0), \lambda, v, t)$ is the subject's current heart rate, which is assumed to depend on time, as well as the initial heart rate $H R(0)$ (as measured at the beginning of the data recording session), the subject's current cardiovascular condition (as defined by the value of $\lambda$ ) and on the velocity $v$ of the exercise,

- $I(t)$ is assumed to be only time-dependent,

- the repelling term $f_{\max }(H R)$ controls the dynamics near the maximum heart rate $H R_{\max }$,

- the repelling term $f_{\min }(H R, \lambda)$ controls the dynamics near the minimum heart rate $H R_{\min }$ and is assumed to depend on $\lambda$,

- the attracting term $f_{D}(H R, H R(0), v, \lambda, t)$ controls the overall heart rate dynamics near the attractor of the heart rate demand $D$ and is assumed to depend on time, velocity $v$, the initial heart rate value $H R(0)$ and the subject's current cardiovascular condition. 
Regarding the minimum heart rate value, it is assumed that $H R_{\min }(\lambda=1)=35$ beats $/$ min for males and $H R_{\min }(\lambda=1)=40$ beats $/$ min for females [24]. It is also assumed that, for $\lambda<1$,

for males and

$$
H R_{\min }(\lambda) \equiv 35 / \lambda \text { beats } / \min
$$

for females [24].

$$
H R_{\min }(\lambda) \equiv(35 / \lambda+5) \text { beats } / \min
$$

The functions $f_{\max }$ and $f_{\min }$ are defined as follows (for more details and an extended study on these functions please refer to [24]):

$$
\begin{gathered}
f_{\max (H R)}=-\left\{1-e^{-\left(\frac{H R-H R_{\max }}{\alpha_{1}}\right)^{2}}\right\} \\
f_{\max (H R \lambda)}=-\left\{1-e^{-\left(\frac{H R-H R_{\min }}{\alpha_{2}}\right)^{2}}\right\}
\end{gathered}
$$

where $\alpha_{1}=\alpha_{2}=10$ beats $/$ min (these optimal numerical values were found by trial and error during a thorough study on fitting the model to time series data [24]).

The function $f_{D}$ is defined as [24]

$$
f_{D}(H R, H R(0), v, \lambda, t) \equiv-\alpha \lambda[H R-D(H R(0), \lambda, v, t)],
$$

where the optimal value of the parameter $\alpha$ was found to be, by trial and error as with the other parameters of the model, $\alpha=0.08 \mathrm{~s}^{-1}$. Assuming that the heart rate demand is affected by the value of $H R(0)$, the total heart rate demand $D$ can be expressed as the sum of a term describing the intensitydependent heart rate demand $D_{d}$ and a term that simulates the heart rate demand "memory" effects [24]:

$D(H R, H R(0), v, \lambda, t)=D_{\mathrm{d}}(v, \lambda, t)+\left\{H R(0)-D_{d}(v, \lambda, t) e^{-\sigma t^{2}}\right\}$.

The standard deviation of the Gaussian-like curve that describes the memory effects on the heart rate demand is modelled [24] by the relation

with optimal parameter values $\alpha_{4}=0.003 \mathrm{~s}^{-2}$ and $\alpha_{5}=4$.

$$
\sigma \equiv \alpha_{4} \lambda\left(\frac{H R_{\max }-H R_{\min }}{H R(0)-H R_{\min }}\right)^{\alpha_{5}}
$$

In the absence of any effects of lactate accumulation (low exercise intensity), the constant value of the heart rate demand $D_{s s}$ is the steady state that the heart rate values reach after a few minutes of exercise. For higher exercise intensities the effects of blood lactate accumulation are apparent a few minutes after the beginning of the exercise and add a "slow component" effect as the exercise continues. This lactate-induced heart rate demand $D_{L a}(\lambda, v, t)$ is assumed to be added as an extra component to $D_{s s}[24]$ :

$$
D_{d}(\lambda, v, t) \equiv D_{s s}(\lambda, v, t)+D_{L a}(\lambda, v, t) \text {. }
$$

For more details on how $D_{L a}(\lambda, v, t)$ is modelled please refer to [24].

For low to moderate intensity exercises, the constant heart rate demand $D_{s s}$ is modelled as follows (see also [12]):

$$
D s s=\frac{v}{v_{\max }}\left(H R_{\max }-H R_{\min }\right)+H R_{\text {rest }}
$$

where the value $\bar{H} R_{\text {rest }}$ refers to the resting heart rate (idling, pre-exercise heart rate for $v=0$ ) and, as discussed in [24], the velocity value $v_{\max } \equiv v_{\max }(\lambda)$ refers to the maximum exercise velocity achievable by an individual of cardiovascular condition $\lambda$. It has been assumed that $v_{\max }(\lambda)$ $=27 \sqrt{\lambda}(\mathrm{km} / \mathrm{h})[24]$.

\section{Intensity pattern simulation}

The simulations were carried out using the mathematical package Maple, version 10 professional.

For the simulations a hypothetical male runner was assumed, with $\lambda=0.85, H R_{\max }=185$ beats $/ \mathrm{min}, H R_{\min }=$ 45 beats/min, a $800 \mathrm{~m}$ running velocity of $25 \mathrm{~km} / \mathrm{h}$ and a velocity $v V O_{2 \max }=20 \mathrm{~km} / \mathrm{h}$ (see also [6]).

Let us denote as $t_{0}$ the exercise (or data recording) starting time.

Aiming to demonstrate the validity as well as the ability of the model [24] to simulate heart rate kinetics, the following different intensity patterns were selected, as examples:

1. incremental exercise, strictly increasing velocity,

2. incremental exercise, sequence of steps,

3. interval training $[4,5]$.

\section{Incremental exercise, strictly increasing velocity}

A velocity function that follows an exponential rise was chosen as an example for this subsection. Denoting as $\tau$ the time constant of the exponential and $v_{m}$ as the maximum velocity value of the training session, then the func-

$$
v(t)=v(m)\left(1-e-\frac{t-t_{0}}{\tau}\right)
$$

tional dependence of the velocity on time, for this specific case, can be expressed as:

For the simulation of this example exercise it was assumed that $t_{0}=0, v_{m}=26 \mathrm{~km} / \mathrm{h}$ and $\tau=12 \mathrm{~s}$. The initial $H R$ value was set equal to $H R(0)=60$ beats $/ \mathrm{min}$ and the total exercise time was set equal to $60 \mathrm{~s}$.

\section{Incremental exercise, sequence of steps}

Let us assume that the velocity pattern in this case consists of a sequence of $N$ steps and let us denote as $T_{s i}$ and $v_{i}$ the time duration and the velocity of the $i$-th step respectively, where $v_{i}<v_{i+1} \forall i=1, \ldots, N-1$.

$$
v(t)=\frac{v_{N}}{2}\left\{1+\frac{1}{N} \sum_{i=1}^{N} \tanh \left(\frac{\left(t-t_{0}\right)-\sum_{j=1}^{i=1} T_{s j}}{\sigma_{\mathrm{S}}}\right)\right\},
$$

The step velocity pattern can be simulated in this case by means of a sum of hyperbolic tangents as follows:

where the parameter $\sigma_{\mathrm{s}}$ is to be numerically chosen so as to simulate the step-wise change in $v$. For the simulations we assumed an example exercise starting from 
$t_{0}=0$, with $v_{i}=5 i \mathrm{~km} / \mathrm{h}, i=1, \ldots 5, T s_{i}=20 \mathrm{~s}$ and total exercise time $100 \mathrm{~s}$. The initial $H R$ value was set equal to $H R(0)=60$ beats $/ \mathrm{min}$. The parameter $\sigma_{\mathrm{s}}$ was chosen to be $\sigma_{\mathrm{s}}=0.5$.

\section{Interval training}

The simulated interval training pattern follows the recommendations presented in [4] and [5]. Let us assume that the velocity pattern consists of a sequence of $N$ sets of exercise intervals and their subsequent recoveries. Let us also denote as $\mathrm{v}_{\mathrm{i}}$ the velocity and $T_{i}$ the time duration of each exercise interval, and similarly $v_{R i}$ the velocity and $T_{R i}$ the time duration of each recovery. If $v_{i} \equiv v_{I}$ and $v_{R i} \equiv v_{R}, \forall i=1, \ldots, N$ then, using a combination of hyper-

$$
\begin{aligned}
v(t)=v_{R} & +\frac{v_{I}-v_{R}}{2} \sum_{i=1}^{N} \tanh \left\{\frac{\left(t-t_{0}\right)-\sum_{j=1}^{i}\left(T_{j}+T_{R j}\right)}{\sigma}\right\}, \\
& +\frac{v_{I}-v_{R}}{2} \sum_{i=1}^{N} \tanh \left\{\frac{-\left(t-t_{0}\right)+\sum_{j=1}^{i-1}\left(T_{j}+T_{R j}\right)+T_{i}}{\sigma}\right\},
\end{aligned}
$$

bolic tangents as before, the velocity pattern of interval training can be simulated by a function given as follows:

As mentioned in the previous subsection, the parameter $\sigma$ is to be numerically chosen. For the simulations presented in the following section, $\sigma=0.5$.

For the purposes of the present subsection, an example anaerobic training session was chosen, consisting of 6 intervals at $125 \% v V O_{2 \max }$, i.e. $v_{I}=25 \mathrm{~km} / \mathrm{h}, v_{R}=0 \mathrm{~km} / \mathrm{h}$ and $T_{i}=T_{R i}=30 \mathrm{~s}$; see $[5,6]$.

The training session was assumed to start at $t=0$ with a $200 \mathrm{~s}$ warm-up exercise at constant velocity of $14 \mathrm{~km} / \mathrm{h}$, from $H R(0)=55$ beats $/ \mathrm{min}$, followed by a $30 \mathrm{~s}$ rest before the interval training. The $30-30$ sessions were thus assumed to start at $t_{0}=230 \mathrm{~s}$.

\section{Details of the numerical procedure}

The resulting simulated heart rate response is provided, in general, via the numerical solution of the two coupled equations of the model [24], i.e. the simultaneous solution of equations (1) and (2).

There are cases, as in the previous sections, where for reasons of simplicity the exercise velocity is provided as an explicit function of time $v(t)$. In such cases the solution of equation (2) is given ab initio and therefore the simulated heart rate time series are given by numerical solution of equation (1) only, where $v \equiv v(t)$.

The numerical solution of equation (1) was carried out in the present study by use of the Runge-KuttaFehlberg (RKF45) method [10], a numerical differential equation solver of order $\mathrm{O}\left(\mathrm{h}^{4}\right)$ with an error estimator of order $\mathrm{O}\left(\mathrm{h}^{5}\right)$. The algorithm's step size was set equal to $\mathrm{h}=0.01$.

\section{Results}

\section{Incremental exercise, strictly increasing velocity}

Figure 1 shows the simulated heart rate time series for the $60 \mathrm{~s}$ incremental exercise of strictly increasing velocity. In the same figure the velocity values are also shown for each time $t$. As can be seen in figure 1, the smooth, slow increase in $H R$, that follows the increase in $v$, was

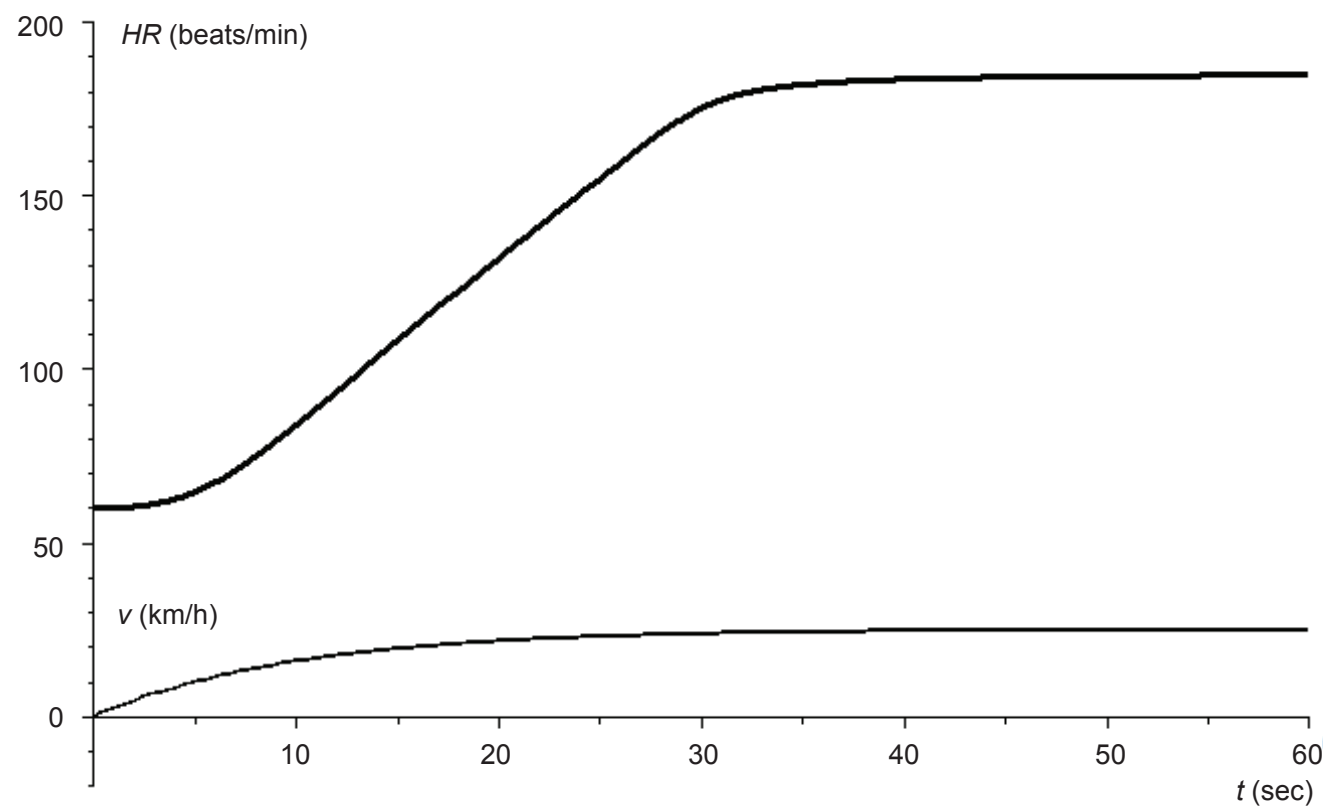

Fig. 1. Incremental exercise, exponentially increasing velocity. On the vertical axis the time series of both the $H R$ and the $v$ are shown (thick and thin black lines respectively) 
successfully simulated. For the interpretation of the plateau $H R$ values towards the end of the exercise it should be remembered that the subject's maximum heart rate was assumed to be $H R_{\max }=185$ beats $/ \mathrm{min}$ and that his $800 \mathrm{~m}$ running velocity was assumed to be $25 \mathrm{~km} / \mathrm{h}$.

\section{Incremental exercise, sequence of steps}

Figure 2 shows the simulated heart rate time series for the $100 \mathrm{~s}$ incremental exercise which consisted of a sequence of steps.
As in the previous case, the $H R$ values follow the stepwise changes in $v$ until $H R_{\max }$ is reached and a plateau in $H R$ values is achieved. By careful observation of figure 2 a short duration "slow component" effect can be seen in the $H R$ time series during the penultimate step, as the $H R$ values approach $H R_{\max }$.

\section{Interval training}

Figure 3 shows the simulated heart rate time series for the $590 \mathrm{~s}$ interval training session.

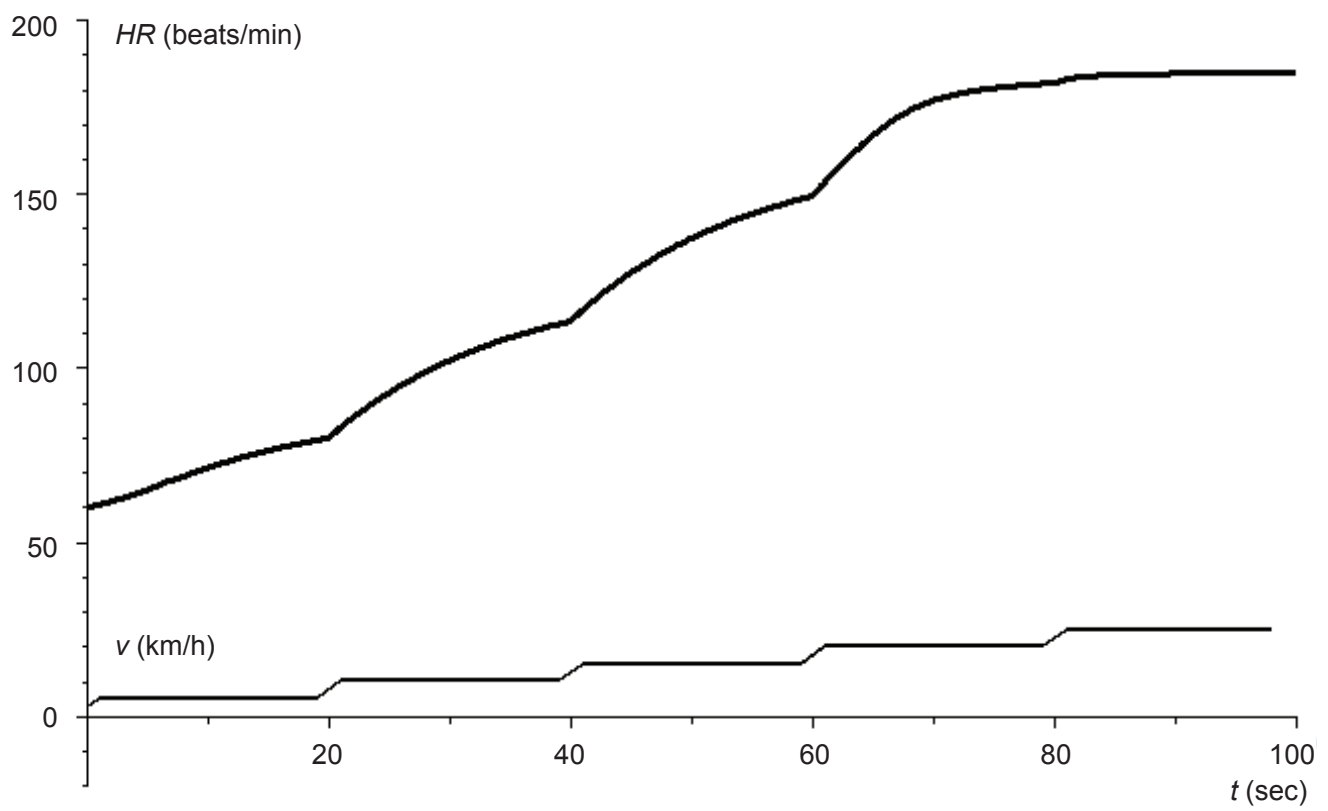

Fig. 2. Incremental exercise, velocity increasing as a sequence of steps. On the vertical axis the time series of both the $H R$ and the $v$ are shown (thick and thin black lines respectively)

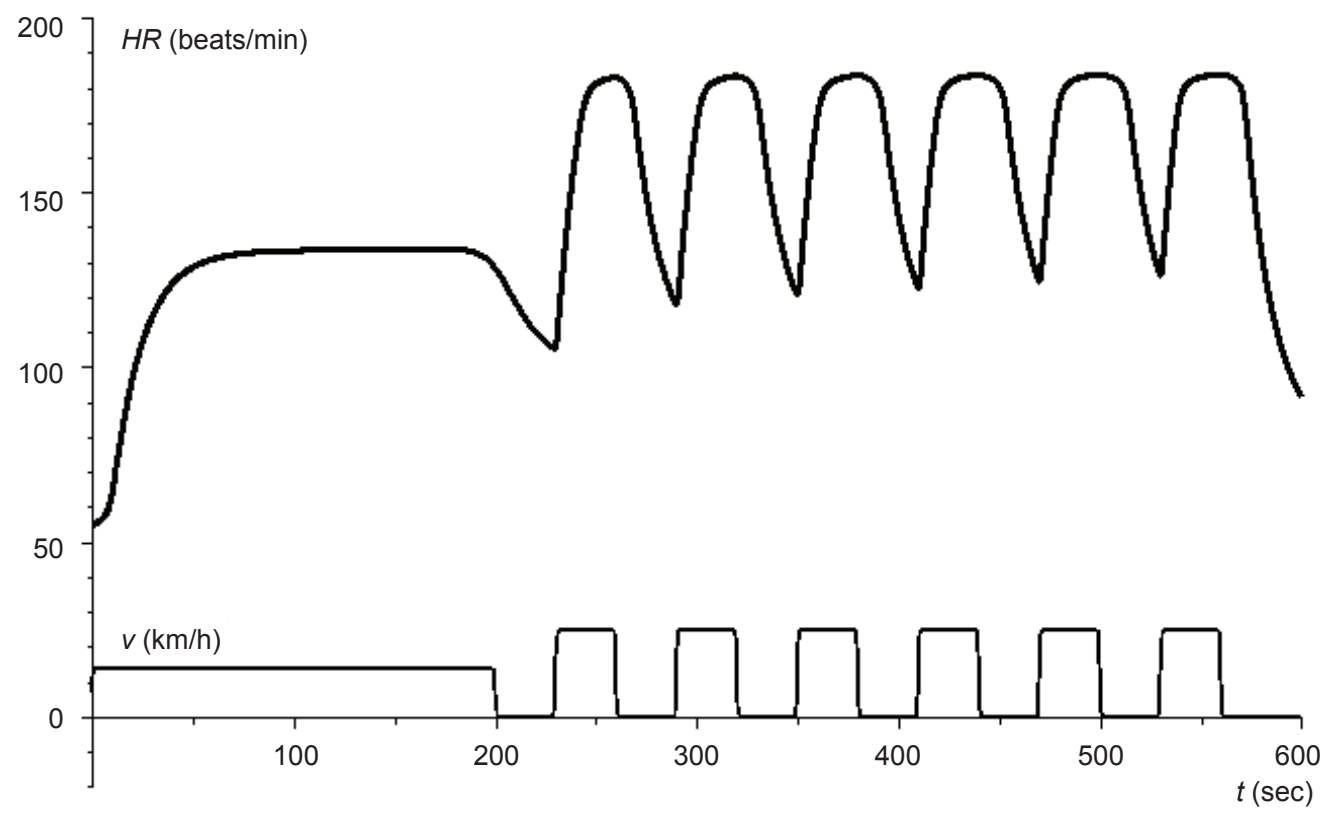

Fig. 3. 30-30 interval training session, following a $200 \mathrm{sec}$ constant velocity warm-up. On the vertical axis the time series of both the $H R$ and the $v$ are shown (thick and thin black lines respectively) 
The success of the model regarding the simulation of such a training session is obvious. of Figure 3 reveals slowly increasing $H R$ recovery values, a phenomenon that can be due to the lactate accumulation/"slow component" effect.

\section{Discussion}

The study of heart rate kinetics is currently the main area of research in exercise physiology. An understanding of heart rate kinetics is fundamental to training methodology and competitive success in sport, as well as to our knowledge of cardiovascular health. A correct and efficient means of interpreting and analyzing heart rate data is of vast importance, as exercise testing is routinely used in both of these areas $[9,16,23]$.

The present work demonstrates the application of a recently proposed dynamical systems model [24] to the simulation of heart rate kinetics during exercises of time-varying intensity. The use of modern mathematical methods of analysis such as the model implemented here would be of much benefit for understanding the relationship between the physiological responses and fitness and/or performance, or for identifying certain features in the physiological time series. As a result, the application of such mathematical methods for analysis and modelling in $H R$ kinetics will have a large impact not only in the development and understanding of training methodology and the testing of athletes but also in the area of medicine. Aspects of general health, recuperation and rehabilitation, as well as diagnosis of many abnormalities in the $H R$ response to movement, or possible disorders that may cause exercise intolerance, are some of the practical advantages of the application of mathematical techniques that model and analyze heart rate responses. Such techniques could also be applied to the study of the heart rate response of population groups for which direct heart rate recordings in intense exercises are not possible or not allowed, such as elderly or pregnant women.

The mathematical model applied in this study is given as a set of coupled ordinary differential equations with respect to time. Its objective is to describe, simulate, fit existing data and ultimately predict the heart rate response to movement. It is worth emphasizing that the power and novelty of this mathematical model lie in the fact that the model is specifically designed (see [24]) to meet the following requirements:

1. A model's features should reflect the body's physiological features.

2. A model should include as few parameters as possible: the model of [24] assumes only one model parameter $(\lambda)$, which reflects the person's overall cardiovascular condition (which improves with appropriate training or rehabilitation). Any changes in this global parameter $(\lambda)$ will reflect improvements in the overall cardiovascular condition (via appropriate training) or loss (due to lack of training or injury). It should be noted here that $0<\lambda<1$, so that the condition of $\lambda=1$ refers to an excellent cardiovascular condition.

3. The functions forming the model should be smooth functions of time.

Regarding comparison of the model implemented herein with other similar existing models, it should be noted that, although the dynamical systems model presented in [20] can be possibly implemented to exercise of linear or non-linear intensities, its fit to raw heart rate time series data has only been demonstrated for exercises of constant intensity [25, 26, 27]. On the other hand, the model of heart rate dynamics during cycling [15] has been successfully applied to heart rate time series data providing numerical values for the model's parameters; however, two more model parameters have been introduced. Finally, even though the improved and advanced version of the model of [20] is capable of following the heart rate dynamics for any linear or nonlinear exercise intensity, its ability to fit heart rate time series data, as well as to simulate and predict heart rate responses, has only been shown for exercises of constant intensity [24].

Regarding the historically first model of cardiovascular kinetics, the 3-phase model, it should be noted that this model emerged as the best fit to the data, from a statistical point of view. Its validity is debatable, as it is very likely that the observed three phases are a figment of the incorrect and overly simple treatment of the data [18]. Furthermore, the parameter values that the model provides can only reflect the body's physiological response to the particular exercise intensity, and no reliable predictions can be made for other exercise intensities. Furthermore, there is a strong interdependency in the model's parameters, which dramatically affects their values and consequently the model's confidence. In comparison, the dynamical systems model applied in the present work is not only able to provide important information regarding the cardiovascular condition of a person, but also to simulate and predict heart rate kinetics for any given exercise intensities.

In the present work, training sessions of exponentially and step-wise increasing velocity have been simulated, as well as an interval training session. The corresponding HR response was also successfully simulated, via application of the mathematical model [24]. Special emphasis should be given to the model's excellent ability to simulate the heart rate response during exercises of complicated 
intensity patterns, such as the interval training session. The study confirms that the applied mathematical model [24] is able not only to provide important information regarding an individual's cardiovascular condition but also to simulate and predict heart rate kinetics for any given exercise intensities.

Future implementations of the model could include fitting to heart rate data of time-varying exercise intensity, where the time series of either $v(t)$ or $\dot{v}=I(t)$ will be known.

\section{References}

1. Astrand P.O., K. Rodahl, H.A. Dahl, S.B. Stromme (2003) Textbook of work physiology - Physiological bases of exercise. Hum. Kinet., 4th edition.

2. Bangsbo J., P. Krustrup, J. Gonzalez-Alonso, R. Bonshel, B. Saltin (2000) Muscle oxygen kinetics at onset of intense dynamic exercise in humans. Am. J. Physiol. Regul. Integr. Comp. Physiol., 279: R899-R906.

3. Barstow T.J., P.A. Mole (1991) Linear and nonlinear characteristics of oxygen uptake kinetics during heavy exercise. J. Appl. Physiol., 71(6): 2099-2106.

4. Billat V.L. (2001) Interval training for performance: a scientific and empirical practice. special recommendations for middle and long distance running. part i: Aerobic interval training. Sports Med., 31(1): 13-31. DOI: 10.2165/00007256-200131010-00002.

5. Billat V.L. (2001) Interval training for performance: a scientific and empirical practice. special recommendations for middle and long distance running. part ii: Aerobic interval training. Sports Med., 31(2): 75-90. DOI: 10.2165/00007256-200131020-00001.

6. Billat V.L., J. Slawinski, V. Bocquet, A. Demarle, L. Lafitte, P. Chassaing, J.P. Ko-ralsztein (2000) Intermittent runs at the velocity associated with maximal oxygen uptake enables subjects to remain at maximal oxygen uptake for a longer time than intense but submaximal runs. Europ. J. Appl. Physiol., 81(3): 188-196. DOI: 10.1007/ s004210050029

7. Bowers R., L. Fox (1992) Sports Physiology. Brown (William C.) Co, 3rd edition.

8. Burke E.R. (1998) Precision Heart Rate Training. Human Kinetics Pub. Inc., 2nd edition.

9. Davies C.T., P.E. Di Prampero, P. Cerretelli (1972) Kinetics of the cardiac output and respiratory gas exchange during exercise and recovery. J. Appl. Physiol., 32: 618-625.

10. Hairer E., S. Norsett, G. Wanner (1993) Solving Ordinary Differential Equations I: Nonstiff Problems. SpringerVerlag, Berlin, 2nd edition. DOI: 10.1007/978-3-54078862-1.
11. Hill A.V., L. Lupton (1923) Muscular exercise, lactic acid and the supply and utilization of oxygen. Q. J. Med., 16: 135-171.

12. Karvonen J., T. Vuorimaa (1988) Heart rate and exercise intensity during sports activities. practical application. Sports Med., 5(5): 303-311, DOI: 10.2165/00007256198805050-00002.

13. Linnarsson D. (1974) Dynamics of pulmonary gas exchange and heart rate changes at start and end of exercise. Acta Physiol. Scand. Suppl., 415: 1-68.

14. Maffetone P. (1996) High Performance Heart: Effective Training with the HRM for Health, Fitness and Competition. Human Kinetics Pub. Inc., 2nd edition.

15. Mazzoleni M.J., C.L. Battaglini, K.J. Martin, E.M. Coffman, B.P. Mann (2016) Modeling and predicting heart rate dynamics across a broad range of transient exercise intensities during cycling. Sports Eng., 19(2): 117-127. DOI: 10.1007/s12283-015-0193-3.

16. Powers S.K., E.T. Howley (1997) Exercise Physiology: Theory and Application to Fitness and Performance. Brown \& Benchmark Publishers, 3rd edition.

17. Sherwood L. (2010) Human Physiology: From Cells to Systems. Brooks/Cole, 7th edition.

18. Stirling J.R., M.S. Zakynthinaki (2009) Counterpoint: The kinetics of oxygen uptake during muscular exercise do not manifest time-delayed phases. J. Appl. Physiol., 107: 1665-1667. DOI: 10.1152/japplphysiol.00158.2009a.

19. Stirling J.R., M.S. Zakynthinaki, V. Billat (2008) Modelling and analysis of the effect of training on $\mathrm{VO}_{2} \mathrm{ki}-$ netics and anaerobic capacity. Bull. Math. Biol., 70(5): 1348-1370. DOI:10.1007/s11538-008-9302-9.

20. Stirling J.R., M.S. Zakynthinaki, I. Refoyo, J. Sampedro (2008) A model of heart rate kinetics in response to exercise. J. Nonlin. Mathemat. Phys., 15(Suppl. 3): 426-436. DOI: 10.2991/jnmp.2008.15.s3.38.

21. Stirling J.R., M.S. Zakynthinaki, B. Saltin (2005) A model of oxygen uptake kinetics in response to exercise: Including a means of calculating oxygen demand/deficit/debt. Bull. Mathemat. Biol., 67(5): 989-1015. DOI: 10.1016/j. bulm.2004.12.005.

22. Whipp B.J., K. Wasserman (1972) Oxygen uptake kinetics for various intensities of constant load work. J. Appl. Physiol., 33: 351-356.

23. Wilmore J., D. Costill (2007) Physiology of sport and exercise. Hum. Kinet., 4th edition

24. Zakynthinaki M.S. (2015) Modelling heart rate kinetics. PLoS ONE, 10(4): e0118263. DOI: 10.1371/journal. pone.0118263.

25. Zakynthinaki M.S., R.O. Barakat, C.A. Cordente Martnez, J. Sampedro Molinuevo (2011) Stochastic optimization for the detection of changes in maternal heart rate kinetics during pregnancy. Comp. Phys. Commun., 182(3): 683-691. DOI: 10.1016/j.cpc.2010.11.027. 
26. Zakynthinaki M.S., J.R. Stirling (2007) Stochastic optimization for modelling physiological time series: application to the heart rate response to exercise. Comp. Phys. Commun., 176(2): 98-108. DOI: 10.1016/j.cpc.2006.08.005.

27. Zakynthinaki M.S., J.R. Stirling (2008) Stochastic optimization for the calculation of the time dependency of the physiological demand during exercise and recovery. Comp. Phys. Commun., 179(12): 888-894. DOI: 10.1016/j.cpc.2008.07.012.
Received 31.08.2016

Accepted 16.11.2016

(C) University of Physical Education, Warsaw, Poland

Acknowledgments

Dedicated to the memory of James Robert Stirling. 